\title{
DEGRADAÇÃO DE CORANTE TÊXTIL ATRAVÉS DE PROCESSO FOTO-FENTON HETEROGÊNEO SOBRE CATALISADOR MAGNÉTICO Nio,2 $\mathrm{Cu}_{0,3} \mathrm{Zn}_{0,5} \mathrm{Fe}_{2} \mathrm{O}_{4}$
}

\author{
F. F. DIAS ${ }^{1 *}$, J. DANTAS ${ }^{2}$, A. CRISTINA ${ }^{2}$, A. S. SILVA ${ }^{2}$, J. N. ANDRADE ${ }^{1}$, J. G. \\ $\mathrm{PACHECO}^{1}$ \\ ${ }^{1}$ Universidade Federal de Pernambuco, Departamento de Engenharia Química \\ ${ }^{2}$ Universidade Federal de Campina Grande, Departamento de Engenharia de Materiais \\ E-mail para contato: *ffsdias@hotmail.com
}

RESUMO - Nesse trabalho foi estudado nanopartículas magnéticas do espinélio $\mathrm{Ni}_{0,2} \mathrm{Cu}_{0,3} \mathrm{Zn}_{0,5} \mathrm{Fe}_{2} \mathrm{O}_{4}$ química e termicamente estável como catalisador na degradação de corante têxtil em processo foto-Fenton heterogêneo. A característica magnética deste material possibilita a facilidade na recuperação do catalisador após o tratamento de efluente aquoso. A literatura pouco relata a utilização desse tipo de material com a finalidade de degradação de compostos tóxicos por geração de radical hidroxila $\left({ }^{\bullet} \mathrm{OH}\right)$ com catalisador heterogêneo. Os testes foram realizados em reator fotoquímico em batelada com lâmpada SunLight (OSRAM) para a fotodegradação do corante têxtil remazol preto $\mathrm{B}$ (RB5) na presença de $\mathrm{H}_{2} \mathrm{O}_{2}$ e catalisador. Foi realizado planejamento fatorial fracionado $\left(3^{3-1}\right)$. Os resultados revelam que 0 melhor resultado foi obtido na condição com $28,7 \mathrm{mmol}$ de $\mathrm{H}_{2} \mathrm{O}_{2} / \mathrm{g}$ de catalisador em $\mathrm{pH} 11$, obtendo-se $96 \%$ de degradação de corante RB5, em 10 minutos de reação.

\section{INTRODUÇÃO}

Os corantes pertencem a uma classe enorme de compostos orgânicos coloridos que representam perigo crescente ao meio ambiente. São observadas perdas de corantes têxteis durante os processos de fabricação e no processo de tingimento de peças de roupas promovendo cor intensa e toxicidade pelo lançamento de águas residuais em sistemas aquáticos (Khataee et al., 2009; Gad-Allah et al., 2009). Quando se trata de resíduos têxteis o problema se torna sério, porque eles são quimicamente e fotoliticamente estáveis, pois são produzidos para serem resistentes ao clima, luz, água e detergente. Esses corantes não são facilmente biodegradáveis em condições aeróbicas apresentam degradação lenta em processos biológicos convencionais de tratamento de efluentes, podendo gerar águas residuais de cores ainda intensas (Guimarães et al., 2012).

O tratamento convencional de efluentes têxteis é muito difícil, porque além de serem tóxicos, variam bastante na sua composição. A aplicação de técnicas de processos oxidativos avançados (POAs) tem mostrado ser eficaz nesse tipo de tratamento (Guimarães et al., 2012; Amorim et al., 2013). Estes processos são baseados na geração de radical hidroxila $\left({ }^{\bullet} \mathrm{OH}\right)$, um poderoso agente oxidante não seletivo que podem reagir com vários tipos de compostos orgânicos, inclusive recalcitrantes (Khataee et al., 2009; Guimarães et al., 2012; Amorim et al., 2013). 
Recentemente tem ocorrido grande interesse na fotocatálise heterogênea aplicada a degradação de poluentes orgânicos por meio de pós semicondutores em nanoescala. Sendo que os fotocatalisadores combinados radiação ultravioleta (UV) vêm ganhado destaque porque geralmente apresentam significativa atividade fotocatalítica (Zhang et al., 2011). Espinélio de ferrita com a fórmula de $\mathrm{MFe}_{2} \mathrm{O}_{4}$, onde $\mathrm{M}$ representa um metal bivalente (como $\mathrm{Cu}, \mathrm{Mn}, \mathrm{Mg}$ e $\mathrm{Zn}$ ) têm atraído bastante atenção devido a suas aplicações potenciais em diversas áreas, incluindo a de fotocatalisadores (Zhang et al., 2011; Ding et al., 2013). Zhang et al. (2011) reportaram em estudo recente que o sistema $\mathrm{MgFe}_{2} \mathrm{O}_{4} / \mathrm{TiO}_{2}$ exibe excelente atividade melhorada na degradação de rodamina B sob radiação UV e irradiação de luz visível melhor do que em presença dos fotocatalisadores $\mathrm{TiO}_{2}$ e P25. Em Ding et al.(2013) foi estudada a degradação do retardante de chama tetrabromobisfenol (TBBPA) utilizando o fotocatalisador magnético $\mathrm{CuFe}_{2} \mathrm{O}_{4}$-Peroximonosulfato, promovendo $99 \%$ de degradação do TBBPA em 30 minutos de reação. Feng et al. (2013) observaram degradação com excelente resultado de atividade catalítica para a redução 4-nitrofenol para 4-aminofenol utilizando nano partículas magnéticas de $\mathrm{CuFe}_{2} \mathrm{O}_{4}$.

No presente trabalho foi estudado a aplicação do espinélio de ferrita do tipo $\mathrm{Ni}_{0,2} \mathrm{Cu}_{0,3} \mathrm{Zn}_{0,5} \mathrm{Fe}_{2} \mathrm{O}_{4}$ (FNiCuZn) como fotocatalisador, com o objetivo de degradação do corante têxtil reativo preto B. A motivação para este estudo está na característica magnética deste catalisador o que permite sua recuperação após o tratamento do efluente, pela aplicação de um campo magnético e a falta de relato do uso de FNiCuZn na literatura.

\section{MATERIAIS E MÉTODOS}

\subsection{Preparação do $\mathrm{Ni}_{0,2} \mathrm{Cu}_{0,3} \mathrm{Zn}_{0,5} \mathrm{Fe}_{2} \mathrm{O}_{4}(\mathrm{FNiCuZn})$}

Para a síntese do FNiCuZn foram utilizados os seguintes reagentes: Nitrato de níquel Hexahidratado $\left(\mathrm{Ni}\left(\mathrm{NO}_{3}\right)_{2} \cdot 6 \mathrm{H}_{2} \mathrm{O}\right)$ Vetec $(\geq 99 \%$, P.M. 145,40), Nitrato de zinco Hexahidratado $\left(\mathrm{Zn}\left(\mathrm{NO}_{3}\right)_{2} .6 \mathrm{H}_{2} \mathrm{O}\right)$ Vetec $(\geq 98 \%$, P.M. 148,73), Nitrato de ferro Nonohidratado $\left(\mathrm{Fe}\left(\mathrm{NO}_{3}\right)_{3} .9 \mathrm{H}_{2} \mathrm{O}\right)$ Vetec ( $\geq 99 \%$, P.M. 808,00), Nitrato de cobre II $\left(\mathrm{Cu}\left(\mathrm{NO}_{3}\right)_{2} .3 \mathrm{H}_{2} \mathrm{O}\right)$ Vetec ( $\geq 99 \%$, P.M. 241,60), Ureia $\left(\mathrm{CO}\left(\mathrm{NH}_{2}\right)_{2}\right)$ Vetec $(\geq 98 \%$, P.M. 60,06$)$.

A síntese dos catalisadores espinélios foi realizada pelo método da combustão com uso de uréia como combustível. A composição inicial da solução foi baseada na valência total dos reagentes oxidantes e redutores utilizando conceitos da química dos propelentes e explosivos (JAIN et al., 1981), de forma que foi estabelecida a estequiometria da fase de interesse. A mistura redox de nitratos metálicos e combustível foi submetida ao aquecimento direto em uma resistência espiral acoplada em uma base cerâmica como suporte. As nanoferritas foram sintetizadas por reação de combustão usando um recipiente com capacidade de produção em bateladas de $10 \mathrm{~g}$. As sínteses das amostras foram realizadas no LabSMaC (Laboratório de Síntese de Materiais Cerâmicos) da UAEMa/UFCG. Os produtos obtidos da combustão foram desaglomerados com auxílio de almofariz e pistilo, passados em peneira com malha 325 mesh (44 $\mu \mathrm{m})$, em seguida encaminhados para as devidas caracterizações. A Figura 1 ilustra o fluxograma da metodologia empregada na síntese da ferrita FNiCuZn. Os catalisadores foram caracterizados por meio das técnicas difração de raios-X, espectroscopia de infravermelho 
médio (FTIR), microscopia eletrônica de varredura, isotermas de adsorção e dessorção de nitrogênio e análise termogravimétrica.

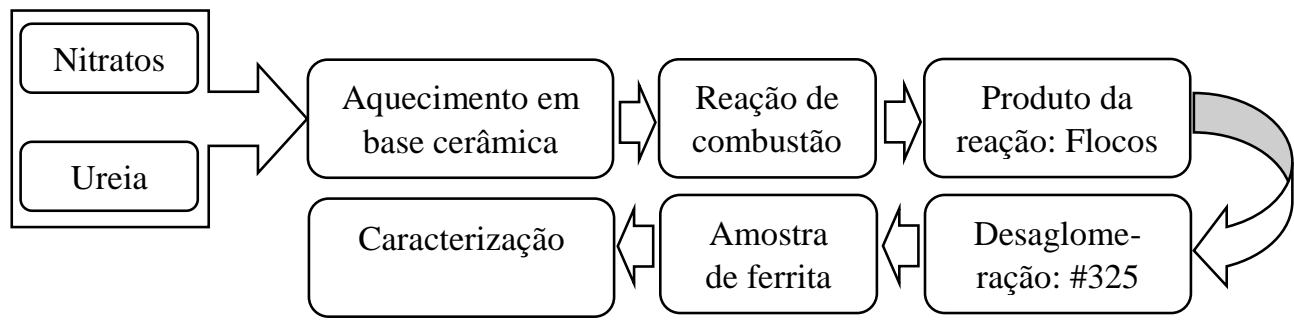

Figura 1 - Fluxograma do processo de obtenção dos pós da ferrita FNiCuZn.

\subsection{Experimento}

Para o teste fotocatalítico, foram utilizados os seguintes materiais e reagentes: corante remazol preto B 133\% (DyStar) (C.I. Reactive Black 5 - RB5 $\left(\mathrm{C}_{26} \mathrm{H}_{21} \mathrm{O}_{19} \mathrm{~N}_{5} \mathrm{~S}_{6} \mathrm{Na}_{4}\right)$, P.M.=986, C.I. 20505, CAS 17095-24-8), peróxido de hidrogênio 30\% $\left(\mathrm{H}_{2} \mathrm{O}_{2}\right)$ Vetec $(\geq 30 \%$, P.M.= 34,02), ácido sulfúrico $\left(\mathrm{H}_{2} \mathrm{SO}_{4}\right) \mathrm{F}$. Maia $(95-98 \%)$, sulfito de sódio $\left(\mathrm{Na}_{2} \mathrm{SO}_{3}\right)$ P.A. ACS ( $\geq 98 \%$, P.M.=126,04), hidróxido de sódio $(\mathrm{NaOH})$ Merck $(\geq 99 \%$, P.M. $=40,0)$, Membrana filtrante Millipore 0,45 $\mu \mathrm{m}$ de $47 \mathrm{~mm}$, Agitador Mecânico IKA RW 20 Digital, lâmpada Osram Sun Light de 300 W, radiômetro UVA/UVB MRU-201.

Para realização dos ensaios, foi feito um planejamento experimental fatorial fracionário de $3^{3-1}$. As variáveis independentes foram a concentração de peróxido de hidrogênio $\left(\mathrm{H}_{2} \mathrm{O}_{2}\right)$, pH e a concentração de FNiCuZn, como pode ser observado na Tabela 1. A variável dependente para análise de respostas será em percentual de degradação de corante RB5, dada pela seguinte equação $\left(1-C / C_{o}\right) \times 100$, sendo $C$ e $C_{o}$ a concentração instantânea e inicial de corante RB5, respectivamente. A degradação do corante RB5 foi medida no decorrer do tempo através de análise em espectrofotômetro UV-Vis modelo Biochrom da série Libra S12 no maior pico de absorção com comprimento de onda igual a $595 \mathrm{~nm}$.

Tabela 1 - Planejamento experimental fatorial fracionário $\left(3^{3-1}\right)$.

\begin{tabular}{|c|c|c|c|}
\hline Ensaio & $\mathrm{H}_{2} \mathrm{O}_{2}(\mathrm{mM})$ & pH & FNiCuZn, $\left(\mathrm{g} \mathrm{L}^{-1}\right)$ \\
\hline 1 & $5(-1)$ & $6(-1)$ & $0,2(-1)$ \\
\hline 2 & $5(-1)$ & $11(1)$ & $0,8(1)$ \\
\hline 3 & $5(-1)$ & $8,5(0)$ & $0,5(0)$ \\
\hline 4 & $39(1)$ & $6(-1)$ & $0,2(-1)$ \\
\hline 5 & $39(1)$ & $11(1)$ & $0,8(1)$ \\
\hline 6 & $39(1)$ & $8,5(0)$ & $0,5(0)$ \\
\hline 7 & $22(0)$ & $6(-1)$ & $0,2(-1)$ \\
\hline 8 & $22(0)$ & $11(1)$ & $0,8(1)$ \\
\hline 9 & $22(0)$ & $8,5(0)$ & $0,5(0)$ \\
\hline 10 & $22(0)$ & $8,5(0)$ & $0,5(0)$ \\
\hline 11 & $22(0)$ & $8,5(0)$ & $0,5(0)$ \\
\hline
\end{tabular}


Para os testes de degradação do corante foram realizados em um reator em batelada com volume de solução igual a $300 \mathrm{~mL}$. O sistema foi mantido sob agitação de $450 \mathrm{rpm}$. A lâmpada utilizada nos testes foi a SunLight Osran de $300 \mathrm{~W}$ que possui o espectro de luz similar ao da luz solar (Figura 2). A radiação medida no sistema com um radiômetro UVA/UVB MRU-201. A concentração de inicial corante RB5 utilizado para todos os testes foi igual a $50 \mathrm{mg} \mathrm{L}^{-1}$. Inicialmente a solução com corante RB5 foi colocada em local escuro sob agitação de 450 rpm por 30 minutos para observar a adsorção do contaminante, isso foi feito em todos os ensaios. Em seguida a solução foi colocada sob a radiação, coletada amostras nos tempos zero, 10, 35 e 60 minutos. A essas amostras foi adicionada solução inibidora de contendo $\mathrm{NaOH}$ e $\mathrm{Na}_{2} \mathrm{SO}_{3}$ para inibir a reação. Em sequência as amostras foram filtradas em membrana filtrante de $0,45 \mu \mathrm{m}$ e armazenada em baixa temperatura.

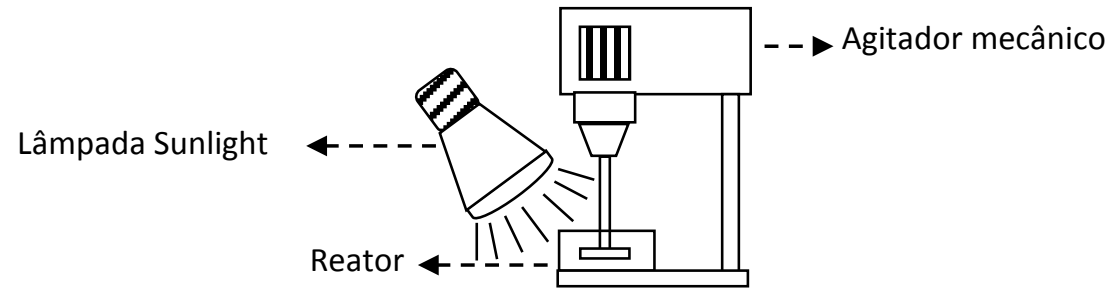

Figura 2 - Esquema fotocatalítico utilizado na degradação de corante RB5.

Os resultados foram analisados estatisticamente e através de observação gráfica através de análise de superfície de resposta para identificação do ponto ótimo de operação do sistema.

\section{RESULTADOS E DISCUSSÃO}

\subsection{Caracterização do $\mathrm{Ni}_{0,2} \mathrm{Cu}_{0,3} \mathrm{Zn}_{0,5} \mathrm{Fe}_{2} \mathrm{O}_{4}$}

Na Figura 3a está ilustrada a curva de difração de raios-X (DRX) da ferrita $\mathrm{Ni}_{0,5} \mathrm{Zn}_{0,5} \mathrm{Fe}_{2} \mathrm{O}_{4}$ (FNiCuZn). A análise revelou a formação apenas da fase espinélio invertido para os sistemas ficha padrão JCPDS 52-0278.
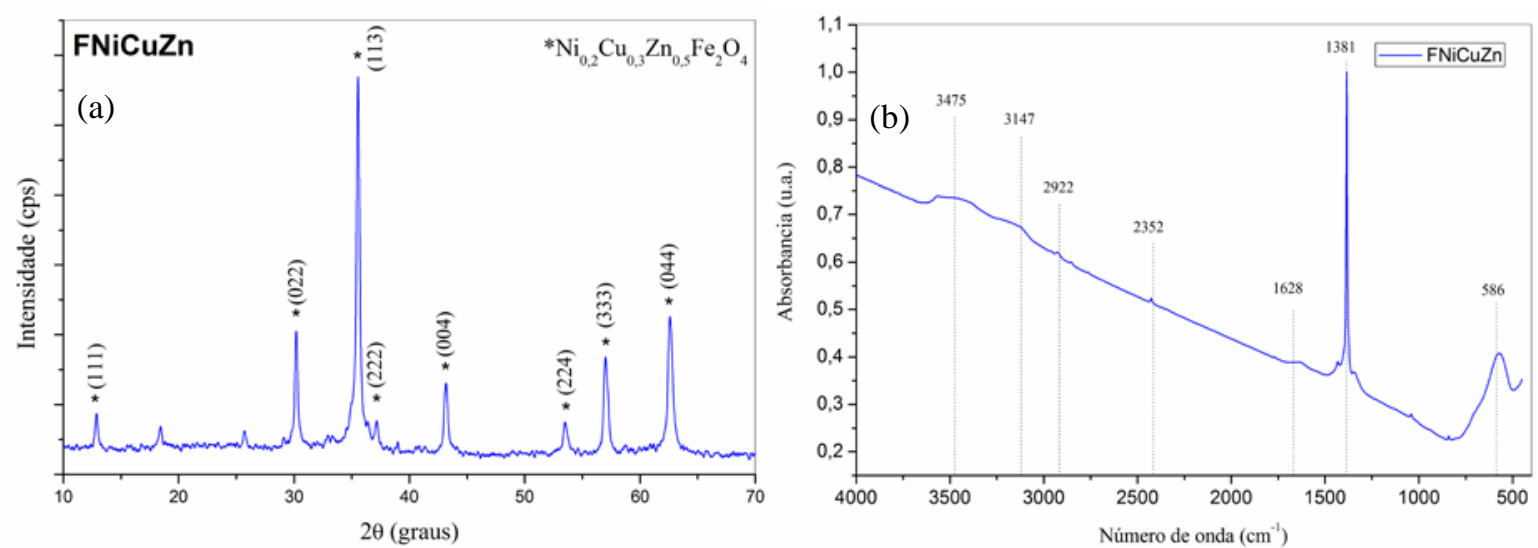

Figura 3 - (a) Difratogramas de raios-X da ferrita FNiCuZn sintetizada por reação de combustão. (b) Espectros de infravermelho FTIR da ferrita FNICuZn. 
Os resultados de espectroscopia de infravermelho médio (FTIR), Figura 3b, verificouse a presença das bandas vibracionais 586, 1381, 1628, 2352, 2922, 3147 e $3475 \mathrm{~cm}^{-1}$ para a amostra. A banda vibracional a $586 \mathrm{~cm}^{-1}$ é atribuída ao estiramento intrínseco de Fe-O no sítio tetraédrico. As demais bandas vibracionais são atribuídas à presença do íon $\mathrm{NO}^{3-}\left(1381 \mathrm{~cm}^{-1}\right)$, a água adsorvida (1628 e $3475 \mathrm{~cm}^{-1}$ ) e resíduo de carbono proveniente do agente complexante (uréia).

Para a ferrita FNiCuZn (Figura 4a), a morfologia é constituída de aglomerados porosos na forma de pequenos blocos, com distribuição de tamanho de 10 a $50 \mu \mathrm{m}$. A porosidade apresentada pelo material se deve em grande parte a liberação dos gases durante a reação de combustão. Observa-se ainda (Figura 4b) que os aglomerados são formados de partículas fracamente ligadas, na forma de placas finas hexagonais.

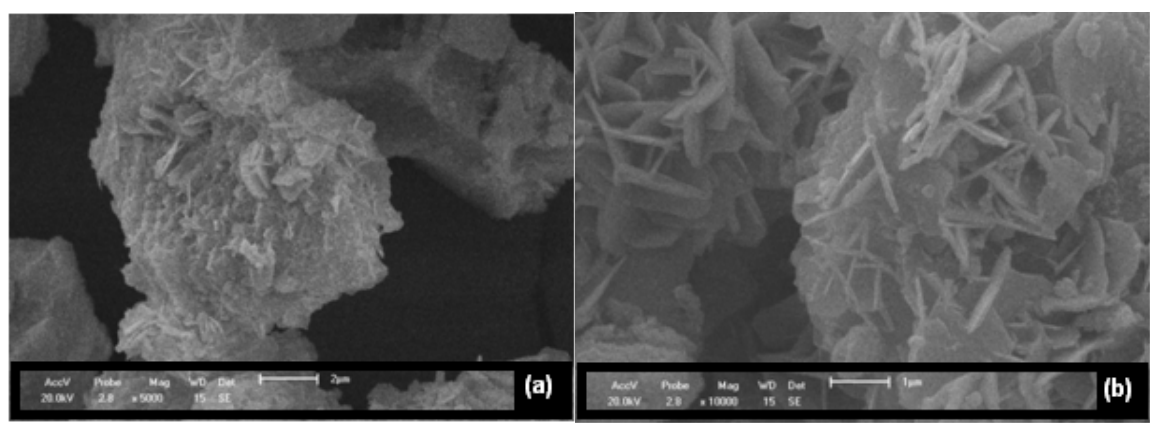

Figura 4 - Microscopia eletrônica de varredura Morfologia do sistema FNiCuZn com aumentos de (a) 5000x e (b) 10000x.

Na Figura 6 apresentam-se as isotermas de adsorção e dessorção de nitrogênio das nano partículas magnéticas (NPMs) de FNiCuZn. Segundo classificação da IUPAC - International Union of Pure and Applied Chemistry, a amostra de FNiCuZn apresenta observa-se perfis de isoterma do tipo IV. De acordo com este tipo de classificação, sugere-se um material com característica mesoporosa com raio de poros variando entre 1 e $25 \mathrm{~nm} 10$ e 250 Å. Também foi observado que a isoterma se apresentou de forma discreta com uma pequena inflexão com pressão relativa (P/P0) de aproximadamente 0,2 , indicando a presença de microporos na estrutura morfológica deste material. A amostra sintetizada apresentou histerese do tipo H3, estando normalmente associado a agregados não rígidos de partículas em forma de placa, originando poros em fenda.

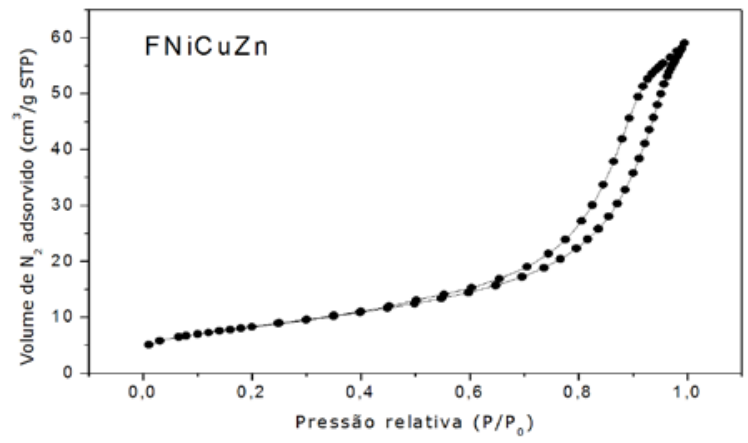

Figura 5 - Isotermas de adsorção e dessorção de nitrogênio do catalisador das NPMs de FNiCuZn. 
Pôde ser observado os valores de área superficial específica total (modelo BET) igual a $29,9 \mathrm{~m}^{2} \mathrm{~g}^{-1}$, volume de poros igual a $0,088 \mathrm{~cm}^{3} \mathrm{~g}^{-1}$ e o diâmetro médio de poros igual a 11,3 nm das NPMs de FNiCuZn.

Por meio da Figura 6, observa-se que as nanopartículas FNiCuZn apresentaram perda de massa em três etapas. A primeira perda de massa ocorre na faixa de temperatura da ambiente a $59^{\circ} \mathrm{C}$, de $0,251 \mathrm{mg}$, equivalente a uma perda de massa percentual de $6,0 \%$, associada a água e gases adsorvidos. A segunda perda de massa ocorre na faixa de $59-295^{\circ} \mathrm{C}$, de $0,227 \mathrm{mg}$ equivalente a uma perda de massa percentual de 5,5\%. A terceira perda de massa ocorre na faixa de $295-913^{\circ} \mathrm{C}$, de $0,077 \mathrm{mg}$, equivalente a uma perda de massa percentual de 1,9\%. Acima de $913^{\circ} \mathrm{C}$ observa-se que a ferrita $\mathrm{FNiCuZn}$ apresentou um patamar de estabilidade térmica.

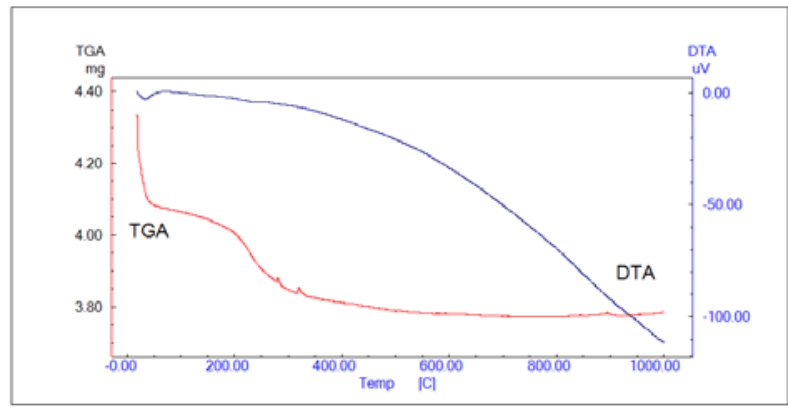

Figura 6 - Curvas termogravimétricas do precursor de FNiCuZn, antes da síntese via combustão, com fluxo de $20 \mathrm{~mL} \mathrm{~min}^{-1}$ ar sintético.

\subsection{Propriedades catalíticas das NPMs do FNiCuZn}

A Tabela 3 mostra o percentual de degradação final em cada experimento, sendo observada a máxima degradação de $94,8 \%$ no teste 8 , que corresponde ao $\mathrm{pH} 11$, máxima concentração de catalisador $\left(0,8 \mathrm{~g} \mathrm{~L}^{-1}\right)$ e concentração intermediária de peróxido (23 mM). A Tabela 4 mostra a análise de variância do modelo matemático obtido em função das variáveis independentes. Os resultados mostram que o modelo se ajusta adequadamente os dados experimentais. O modelo matemático obtido é dada pela Equação 1. A tabela 3 mostra pequenos erros entre os valores experimentais observados e os valores preditos pelo modelo. Na Figura 7 pode ser observado o percentual de degradação do corante RB5 em um gráfico de superfície de resposta do processo $\mathrm{FNiCuZn} / \mathrm{H}_{2} \mathrm{O}_{2} / \mathrm{UV}$-Vis. A concentração de FNiCuZn que ocorre maior degradação de corante RB5 em pH 11 e concentração de peróxido igual 23 mM.

$\left(\mathbf{1}-\mathbf{C} / \boldsymbol{C}_{\boldsymbol{o}}\right) \mathbf{x} \mathbf{1 0 0}=202,108( \pm 6,713)+1,656( \pm 0,129) \cdot\left[\mathrm{H}_{2} \mathrm{O}_{2}\right]-0,0233( \pm 0,0020) \cdot\left[\mathrm{H}_{2} \mathrm{O}_{2}\right]^{2}-$ $45,108( \pm 1,602) \cdot[p H]+3,118( \pm 0,092) \cdot[p H]^{2}-0,0516( \pm 0,0108) \cdot[p H] \cdot\left[\mathrm{H}_{2} \mathrm{O}_{2}\right]$

Na Figura 7 pode ser observado ainda a simulação na degradação de corante em 10 minutos de reação e uma concentração de FNiCuZn igual a $0,8 \mathrm{~g} \mathrm{~L}^{-1}$, variando o pH de 6 a 11 e de peróxido de hidrogênio de 5 a 39 mM. Sendo observado máxima degradação de corante 
RB5 em pH 11 e concentração de peróxido de hidrogênio igual a $23 \mathrm{mM}$. Assim, foi obtido uma razão de peróxido de hidrogênio/catalisador igual a 28,8 mmol de $\mathrm{H}_{2} \mathrm{O}_{2} / g$ de FNiCuZn.
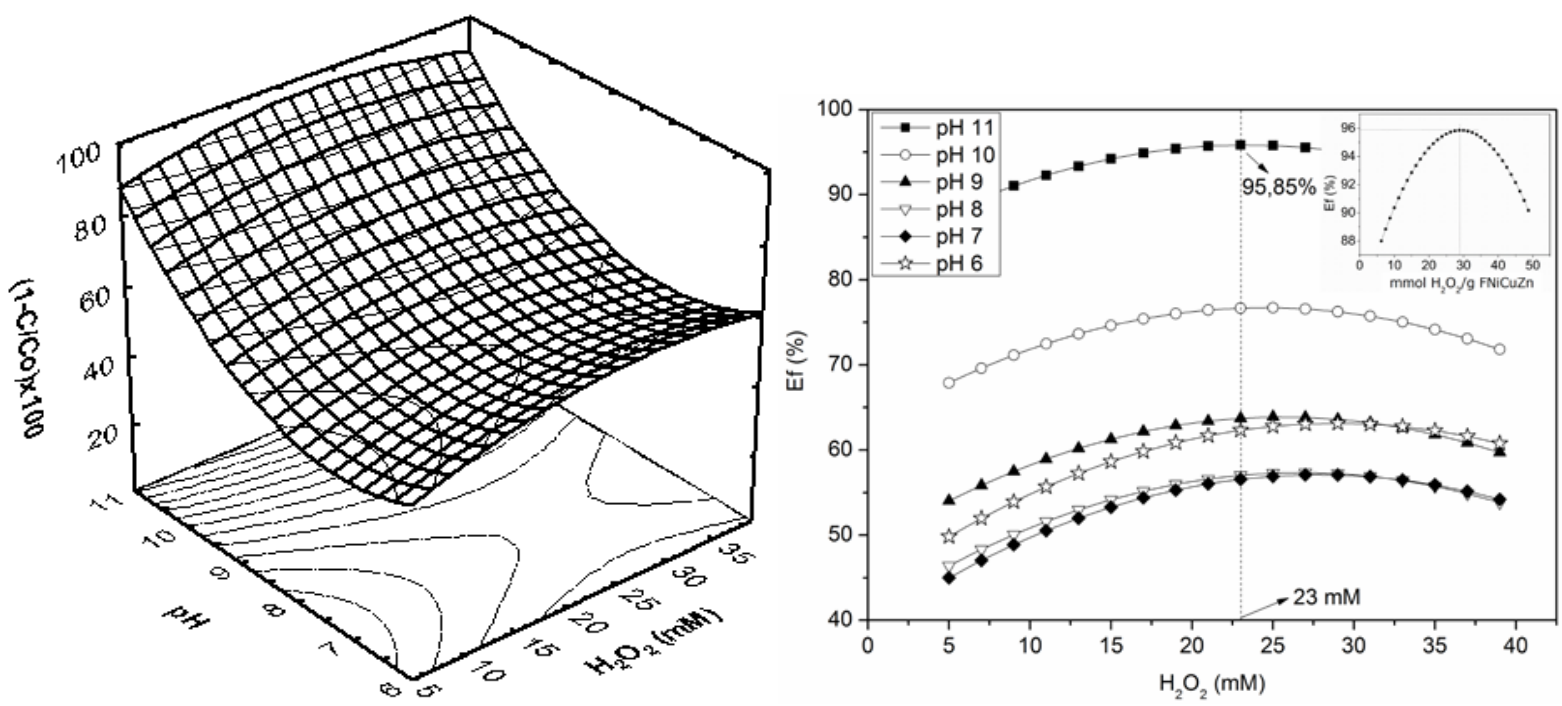

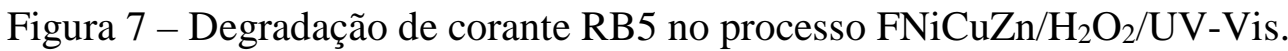

Na Tabela 2 pode ser visto os valores observados experimentalmente e os valores calculados pelo modelo matemático da Equação 1. Pode ser visto ainda que o resíduo é baixo mostrando bom ajuste do modelo.

Tabela 2 - Condições experimentais e resultados de degradação percentual de corante RB5 com observados e preditos pelo modelo matemático e o resíduo.

\begin{tabular}{c|c|c|c}
\hline Ensaios & Observado & Predito & Resíduo \\
\hline 1 & 48,42 & 49,84 & $-1,42$ \\
2 & 89,00 & 88,01 & 0,99 \\
3 & 49,87 & 49,44 & 0,43 \\
4 & 58,40 & 60,79 & $-2,39$ \\
5 & 90,20 & 90,18 & 0,03 \\
6 & 58,36 & 56,00 & 2,36 \\
7 & 65,85 & 62,04 & 3,81 \\
8 & 94,80 & 95,82 & $-1,01$ \\
9 & 57,67 & 59,44 & $-1,78$ \\
10 & 58,37 & 59,44 & $-1,07$ \\
11 & 59,50 & 59,44 & 0,05 \\
\hline
\end{tabular}

Um modelo cinético de pseudo-primeira-ordem para a degradação do corante RB5 próximo da condição otimizada foi obtido pela linearização da equação $\ln \left(C / C_{o}\right)=-k_{a p} \times t$, sendo $k_{a p}$ a constante de velocidade aparente, e $t$ o tempo de reação em segundos. Sendo a constante cinética ajustada $K_{a p}$ igual a $1,2 \times 10^{-3} \mathrm{~s}^{-1}$. Em trabalho relatado por Feng et al. (2013) utilizando nano partículas de $\mathrm{CuFe}_{2} \mathrm{O}_{4}$ para redução de nitrofenol foi obtido um $k_{a p}$ de 
$1,2 \times 10^{-1} \mathrm{~s}^{-1}$. Testes realizados por Li et al. (2012) com nano compósitos de $\mathrm{Fe}_{3} \mathrm{O}_{4} /$ grafeno/Pt e $\mathrm{Fe}_{3} \mathrm{O}_{4} /$ grafeno/Pd na redução de 4-nitrofenol foram obtidos $k_{a p}$ iguais a $6,1 \times 10^{-2} \mathrm{~s}^{-1}$ e $8,8 \times 10^{-3}$ $\mathrm{s}^{-1}$, respectivamente.

\section{CONCLUSÕES}

O espinélio cristalino $\mathrm{Ni}_{0,2} \mathrm{Cu}_{0,3} \mathrm{Zn}_{0,5} \mathrm{Fe}_{2} \mathrm{O}_{4}$ foi obtido pelo método de síntese por combustão. O uso deste material em processo do tipo foto-Fenton heterogêneo com radiação solar artificial apresentou 95\% de degradação do corante RB5 em pH 11, concentração de catalisador $0,8 \mathrm{~g} \mathrm{~L}^{-1}$ e concentração de peróxido iguala a $23 \mathrm{mM}$. Esse material possui propriedades magnéticas que pode facilitar sua recuperação após o tratamento do efluente. Assim o $\mathrm{Ni}_{0,2} \mathrm{Cu}_{0,3} \mathrm{Zn}_{0,5} \mathrm{Fe}_{2} \mathrm{O}_{4}$ possui potencial de aplicação no tratamento de efluentes tóxicos industriais com uso de radiação solar em meio alcalino, sem a necessidade de acidificar o efluente.

\section{REFERÊNCIAS}

AMORIM, C. C.; LEÃO, M. M. D.; MOREIRA, R. F. P. M.; FABRIS, J. D.; HENRIQUES, A. B. Performance of blast furnace waste for azo dye degradation through photo-Fenton-like processes. Chem. Eng. J., v. 224, p. 59-66, 2013.

DING, Y.; ZHU, L.; WANG, N.; TANG, H. Sulfate radicals induced degradation of tetrabromobisphenol A with nanoscaled magnetic $\mathrm{CuFe}_{2} \mathrm{O}_{4}$ as a heterogeneous catalyst of peroxymonosulfate. Appl. Catal. B: Environ., v. 129, p.153-162, 2013.

FENG, J.; SUA, L.; MA, Y.; REN, C.; GUO, Q.; CHEN, X. $\mathrm{CuFe}_{2} \mathrm{O}_{4}$ magnetic nanoparticles: A simple and efficient catalyst for the reduction of nitrophenol. Chem. Eng. J., v. 221, p. 1624, 2013.

GAD-ALLAH, T. A.; KATO, S.; SATOKAWA, S.; KOJIMA, T. Treatment of synthetic dyes wastewater utilizing a magnetically separable photocatalyst $\left(\mathrm{TiO}_{2} / \mathrm{SiO}_{2} / \mathrm{Fe}_{3} \mathrm{O}_{4}\right)$ : Parametric and kinetic studies. Desalination, v. 244, p. 1-11, 2009.

GUIMARÃES, J. R.; MANIERO, M. G.; ARAÚJO, R. N. A comparative study on the degradation of RB-19 dye in an aqueous medium by advanced oxidation processes. $J$. of Environ. Manage., v. 110, p. 33-39, 2012.

KHATAEE, A. R.; PONS, M. N.; ZAHRAA, O. Photocatalytic degradation of three azo dyes using immobilized $\mathrm{TiO}_{2}$ nanoparticles on glass plates activated by UV light irradiation: Influence of dye molecular structure. J. Hazard. Mat., v. 168, p. 451-457, 2009.

Li, X. Y.; Wang, X.; Song, S. Y.; Liu, D. P.; Zhang, H. J. Selectively deposited noble metal nanoparticles on $\mathrm{Fe}_{3} \mathrm{O}_{4} /$ graphene composites: stable, recyclable, and magnetically separable catalysts. Chem.-Eur. J. v. 18, p. 7601-7607, 2012.

ZHANG, L.; HE, Y.; WU, Y.; WU, T. Photocatalytic degradation of RhB over $\mathrm{MgFe}_{2} \mathrm{O}_{4} / \mathrm{TiO}_{2}$ composite materials. Mat. Sci. and Eng. B, v. 176, p. 1497- 1504, 2011. 